\title{
Intravenous administration of DPSCs and BDNF improves neurological performance in rats with focal cerebral ischemia
}

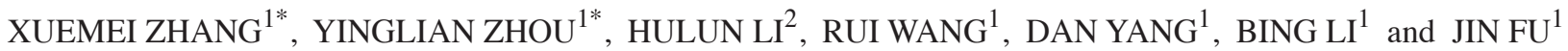 \\ ${ }^{1}$ Department of Neurology, The Second Affiliated Hospital of Harbin Medical University; ${ }^{2}$ Department of Neurobiology, \\ Neurobiology Key Laboratory, Harbin Medical University, Harbin, Heilongjiang 150086, P.R. China
}

Received December 7, 2016; Accepted February 12, 2018

DOI: $10.3892 / \mathrm{ijmm} .2018 .3517$

\begin{abstract}
Dental pulp stem cells (DPSCs) are considered as an ideal stem cell source for the treatment of neurological diseases. In this study, we evaluated the therapeutic potency of DPSCs and brain-derived neurotrophic factor (BDNF) in focal cerebral ischemia using animal models. Following middle cerebral artery occlusion (MCAO), rats were randomized into four groups: the BDNF, DPSCs, DPSCs+BDNF and the controls injected with saline. DPSCs were transplanted and BDNF was injected into the DPSCs+BDNF group via the tail vein. The fate of the transplanted DPSCs in rat brains was evaluated using immunofluorescence, immunohistochemistry, western blot analysis and reverse transcription-polymerase chain reaction (RT-PCR). Adhesive removal tests and the modified neurological severity scores were used to estimate the restoration of neurological function. Proliferation of intravenously transplanted DPSCs was observed in the peripheral ischemic regions of the MCAO models. A green fluorescent dye PKH67 was used to label cells. PKH67-labeled DPSCs were co-localized with neuronal cell markers and 4',6-diamidino-2-phenylindole (DAPI). DPSC transplantation combined with BDNF induced the expression of neural differentiation markers such as nestin, doublecortin (DCX) and neuronal specific filament (NF-H), suggesting that BDNF enhances the survival of DPSCs and differentiation into neuronal cells. Treatment with DPSCs combined with BDNF promoted the recovery of neurological function more effectively compared with BDNF injection or DPSC transplantation alone. In conclusion, treatment with DPSCs combined with BDNF enhances neurological recovery after stroke suggesting a novel therapeutic strategy against cerebral ischemia.
\end{abstract}

Correspondence to: Dr Jin Fu, Department of Neurology, The Second Affiliated Hospital of Harbin Medical University, 246 Xuefu Road, Nangang, Harbin, Heilongjiang 150086, P.R. China

E-mail: fujin198@126.com

*Contributed equally

Key words: dental pulp stem cells, brain derived neurotrophic factor, cell transplantation

\section{Introduction}

Ischemic stroke is a devastating neurological disorder and is one of the leading causes of death worldwide. It entails heavy financial burden for patients and their families, and society. Treatment is limited to interventions such as thrombolysis, anticoagulation and antiplatelet therapies. Neuroprotective treatments have been reported to restore neurological function (1). Stem cell transplantation is a promising strategy to restore neurological function after stroke. Dental pulp stem cells (DPSCs) trigger tissue regeneration and differentiation into cells such as osteoblasts, odontoblast-like cells, adipocytes, and neurons. Gronthos and his colleagues first isolated DPSCs from human dental pulp in 2000 (2). DPSCs represent adult multipotent cells originating in the cranial neural crest. They secrete neurotrophic factors. Previous studies suggest that human DPSCs potentially differentiate into functional neural progenitors or neurons in vitro, which integrate into rat brains in vivo, playing a therapeutic role in brain and spinal cord injury, and neurodegenerative diseases (3-7).

However, a major challenge relates to the limited viability and neuronal differentiation in vivo. Brain-derived neurotrophic factor (BDNF) is a member of the neurotrophin growth factor family that includes nerve growth factor (NGF), neurotrophin-3 (NT-3), and neurotrophins-4/5 (NT-4/5) (8). BDNF acts via high-affinity receptor tyrosine kinase B (TrkB), expressed in the central nervous system (9). As reported previously, BDNF exerts trophic effects on cortical, hippocampal and cerebellar neurons in vitro. BDNF protects motor neurons, hippocampal, and substantia nigra dopaminergic neurons against brain injury. It plays a vital role in neuronal growth, differentiation, and migration (10). Ischemic brain injury triggers BDNF expression, as part of neuroprotective response (11). Intraventricular injection of BDNF before focal cerebral ischemia (12), intracerebral infusion of BDNF following stroke (13), and transplantation of BDNF-treated mesenchymal or neural stem cells alleviated tissue damage in the brain and improved behavioral recovery (14-16).

However, studies investigating the fate of transplanted DPSCs in the injured brain and their neuroprotective function when combined with BDNF are limited. We propose that DPSCs and BDNF are neuroprotective in experimental mouse models of cerebral ischemia, and restore neurological function after stroke. In this study, we determined the fate of transplanted DPSCs in the rat brain. Additionally, we investigated 
whether intravenous administration of rat-derived DPSCs enhanced neurological outcomes after ischemic stroke. We demonstrated that BDNF combined with DPSCs increased the viability of transplanted cells, facilitated the differentiation and neuroprotective effects compared with DPSCs or BDNF alone.

\section{Materials and methods}

Experimental design. We randomized the rats into four groups immediately after middle cerebral artery occlusion (MCAO) (Fig. 1). Rats were anesthetized via intraperitoneal injection with $10 \%$ chloral hydrate $(0.35 \mathrm{ml} / 100 \mathrm{~g}) 24 \mathrm{~h}$ after stroke. The control group was treated with $0.3 \mathrm{ml}$ saline as vehicle during the subacute phase. Animals in the BDNF group were treated with $0.3 \mathrm{ml} \mathrm{BDNF}(50 \mu \mathrm{g} / \mathrm{ml})$ injection via the tail vein. Rats in the DPSCs group were injected with $0.3 \mathrm{ml}$ DPSCs suspension $\left(1 \times 10^{7} / \mathrm{ml}\right)$ via the tail vein very slowly. Rats in the DPSCs+BDNF group were injected with $0.3 \mathrm{ml}$ DPSCs $\left(1 \times 10^{7} / \mathrm{ml}\right)$ and $\operatorname{BDNF}(50 \mu \mathrm{g} / \mathrm{ml})$ via the tail vein.

Isolation, identification, in vitro fluorescent labeling. DPSCs were obtained from the incisors of 4-week-old male Sprague-Dawley (SD) rats under sterile conditions as previously described (17). In brief, dental pulp was treated with $3 \mathrm{mg} / \mathrm{ml}$ collagenase type I at $37^{\circ} \mathrm{C}$ for $1 \mathrm{~h}$ with gentle shaking, followed by centrifugation (1,500 rpm for $5 \mathrm{~min}$ ). The supernatant and enzymes were discarded, and the residue was cultured in $\alpha$-modified Eagle's medium ( $\alpha$-MEM) containing penicillin $(1 \%, 100 \mathrm{U} / \mathrm{ml})$, streptomycin $(1 \%, 100 \mu \mathrm{g} / \mathrm{ml})$ and fetal bovine serum (FBS 10\%; Gibco, Grand Island, NY, USA) at $37^{\circ} \mathrm{C}$ and $5 \% \mathrm{CO}_{2}$. The medium was replaced every 3 days. Cells at $80 \%$ confluency were passaged. DPSCs were identified by flow cytometry based on the surface markers on stem cells. In brief, $\sim 1 \times 10^{6}$ cells were incubated with $2 \%$ FBS in phosphate-buffered saline (PBS) at $4^{\circ} \mathrm{C}$ for $30 \mathrm{~min}$ and $1 \mu \mathrm{l}$ of rat monoclonal antibody specific for Sca-1, CD34, CD44 (18) and CD45 (19). Cells in the control experiment were cultured in buffer solution without primary antibodies. DPSCs were pre-labeled with green fluorescent dye PKH67 up to 90\%. The final density of the labeled cell suspension was $\sim 1 \times 10^{7}$ cells $/ \mathrm{ml}$.

Induced differentiation. To test the pluripotency of the isolated DPSCs, passage 3 cells were seeded in 6-well culture plates up to 20,000 cells $/ \mathrm{cm}^{2}$. The proliferation medium was replaced by the inductive medium. For mineralized nodules, the differentiation-inducing medium was supplemented with ascorbic acid, dexamethasone, and an excess of phosphate. Osteogenesis was promoted by supplementing $50 \mathrm{~g} / \mathrm{ml}$ ascorbic acid 2-phosphate, $10 \mathrm{~nm}$ dexamethasone and $10 \mu \mathrm{M} \beta$-glycerolphosphate. Adipogenesis was induced with $100 \mathrm{~nm}$ dexamethasone and $50 \mu \mathrm{g} / \mathrm{ml}$ indometacin. The culture was maintained for 3 weeks, during which the medium was changed every 2-3 days. Finally, the cells were stained with Alizarin Red and Oil Red O, and visualized under light microscopy.

Focal cerebral ischemia. The animal model of focal cerebral ischemia was generated by transient occlusion of the right middle cerebral artery (MCA) (20). Adult male SD rats weighing $250 \pm 10 \mathrm{~g}$ each, were purchased from the Animal Center of the Second Affiliated Hospital of Harbin Medical University. All the procedures were approved by the Ethics Committee of the Harbin Medical University, and were compliant with the NIH guidelines for use and care of laboratory animals. In brief, rats were anesthetized with $10 \%$ chloral hydrate $(0.35 \mathrm{ml} / 100 \mathrm{~g})$. A midline cervical incision was used to dissect the right common carotid artery (CCA), external carotid artery (ECA) and internal carotid artery (ICA). A 4-0 monofilament nylon thread with a silicone-coated tip (diameter $0.37 \mathrm{~mm}$, length 3-4 $\mathrm{mm}$ ) was extended from the ECA into the ICA to mask the origin of the right MCA. The nylon thread was secured and the incision was closed. The rat body temperature was maintained at $37 \pm 1^{\circ} \mathrm{C}$ using an incandescent lamp. Two hours later, the thread was withdrawn to perform reperfusion. Sham-operated animals were subjected to similar procedures without occlusion of the right MCA. MCAO was confirmed by suspending the rats by the tail for contralateral forelimb flexion. Further, circling behavior on the floor was detected. The weight of rats after ischemia was recorded at $0,1,7,14$ and 28 days post-MCAO.

2,3,5-Triphenyltetrazolium chloride (TTC) staining. TTC staining was used to verify ischemic injury at $24 \mathrm{~h}$ post-MCAO. The brain wa rapidly removed on ice under anesthesia. Fresh brains were frozen for $25 \mathrm{~min}$ at $-20^{\circ} \mathrm{C}$, sliced into five consecutive coronal sections, incubated in 2\% TTC (Sigma-Aldrich, St. Louis, MO, USA) at $37^{\circ} \mathrm{C}$ for $20 \mathrm{~min}$ in the dark. Healthy zones appeared red and infarcted tissues were white in color. Brain sections were photographed using a digital camera. The infarct volume was determined as a percentage of dead cerebral tissue.

Histological examination. The brain tissues from rats were embedded in paraffin. Serial coronal sections were produced and mounted on slides. The specimens were stained with hematoxylin and eosin (H\&E) according to standardized protocols, and visualized by light microscopy.

Adhesive removal test and modified neurological severity scores ( $m N S S$ ). Two small pieces of adhesive-backed paper dots, measuring $113.1 \mathrm{~mm}^{2}$ in size, were used as bilateral tactile stimuli to the distal-radial region of the wrist in each forelimb. Rats were pre-trained for a week before MCA occlusion. They were monitored on days 1, 7, 14, 21 and 28 after stroke. The mean duration of the three trials for the removal of adhesive papers was recorded (21). The mNSS encompass a series of motor, sensory, balance and reflex tests graded on a scale of 0-18 (normal 0; maximal deficit score 18). Scores reflect the degree of inability to perform a specific test: higher scores suggest severe neurological deficit (22).

Immunofluorescence. The rats were anesthetized with $10 \%$ chloral hydrate $0.35 \mathrm{ml} / 100 \mathrm{~g}$ and their chests were split to expose the hearts. The animals were rapidly perfused with $400 \mathrm{ml}$ saline, followed by $300 \mathrm{ml} 4 \%$ paraformaldehyde (pH 7.4) through the left ventricle. The brain tissues of the rats were post-fixed in $4 \%$ paraformaldehyde $\left(\mathrm{pH} \mathrm{7.4)}\right.$ at $4^{\circ} \mathrm{C}$ for $24 \mathrm{~h}$. Brains were dehydrated in a graded series of sucrose at 20 and $30 \%$ daily. Frozen sections of the brain were blocked with normal goat serum for $20 \mathrm{~min}$ at room temperature, and incubated with anti-nestin (1:100), anti-doublecortin (DCX) (1:100), and anti-neuronal specific filament (NF-H) (1:200) overnight at $4^{\circ} \mathrm{C}$. They were washed with PBS three times $(5$ min each 
Table I. The antibodies.

\begin{tabular}{|c|c|c|c|c|c|c|}
\hline Antibody & Conjugation & Manufacturer & $\begin{array}{c}\text { Dilution } \\
\text { (FACS) }\end{array}$ & $\begin{array}{l}\text { Dilution } \\
\text { (IF) }\end{array}$ & $\begin{array}{l}\text { Dilution } \\
\text { (IHC) }\end{array}$ & $\begin{array}{l}\text { Dilution } \\
\text { (WB) }\end{array}$ \\
\hline Sca-1 & FITC & Biolegend & $1: 100$ & & & \\
\hline CD34 & APC & Biolegend & $1: 100$ & & & \\
\hline CD44 & PE & Biolegend & $1: 100$ & & & \\
\hline CD45 & Percp & Biolegend & $1: 100$ & & & \\
\hline Nestin & & Abcam & & $1: 100$ & $1: 200$ & $1: 1,000$ \\
\hline DCX & & Abcam & & $1: 100$ & $1: 200$ & $1: 500$ \\
\hline $\mathrm{NF}-\mathrm{H}$ & & Abcam & & $1: 200$ & $1: 200$ & $1: 1,000$ \\
\hline Goat anti-rabbit & Rhodamine & Zhongshan & & $1: 100$ & & \\
\hline Goat anti-mouse & Rhodamine & Zhongshan & & $1: 100$ & & \\
\hline Goat anti-rabbit & HRP & Zhongshan & & & $1: 400$ & \\
\hline Goat anti-mouse & HRP & Zhongshan & & & $1: 400$ & \\
\hline Goat anti-rabbit & HRP & Zhongshan & & & & $1: 5,000$ \\
\hline Goat anti-mouse & HRP & Zhongshan & & & & $1: 5,000$ \\
\hline
\end{tabular}

FACS, fluorescence-activated cell sorting; NF-H, neuronal specific filament; HRP, horseradish peroxidase; DCX, doublecortin.

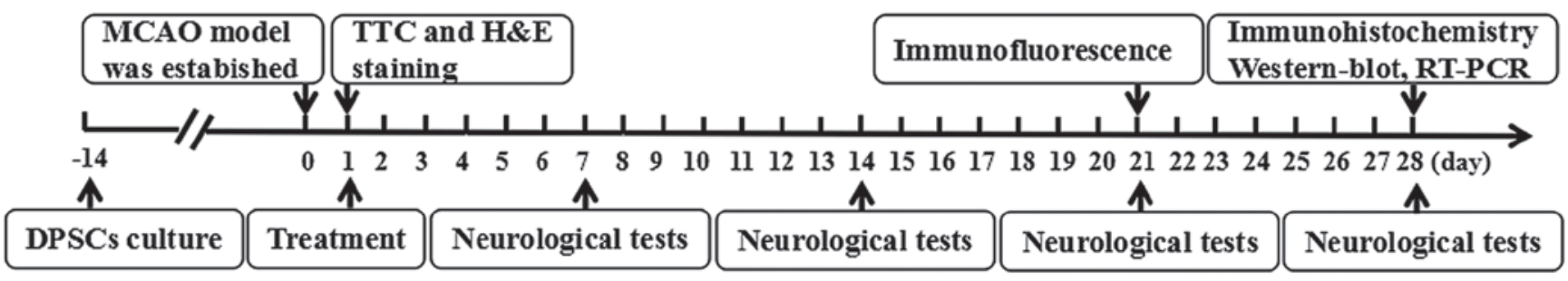

Figure 1. Dental pulp stem cells (DPSCs) were isolated and cultured for 14 days before the middle cerebral artery occlusion (MCAO) models were established. The four groups of rats were treated with saline, brain-derived neurotrophic factor (BDNF) injection, DPSCs transplantation, DPSC transplantation combined with BDNF injection, respectively, 24 h post-MCAO. Behavioral tests were conducted on days 1, 7, 14, 21 and 28 postoperatively. Immunofluorescence was conducted on day 21. Immunohistochemistry, western blot analysis and RT-PCR were performed on day 28.

time) followed by $1 \mathrm{~h}$ reaction with rhodamine-conjugated goat anti-rabbit and anti-mouse IgGs. Brain sections were counterstained with DAPI for $10 \mathrm{~min}$ at $37^{\circ} \mathrm{C}$. The survival and spatial distribution of PKH67-labeled DPSCs were observed using a laser scanning confocal microscope. Five randomly-selected fields were used to count the average number of positive cells in the regions. The antibodies are listed in Table I.

Immunohistochemistry. Paraffin sections were washed with xylene and ascending alcohol, to air-dry and hydration, and incubated in $1 \% \mathrm{H}_{2} \mathrm{O}_{2}$ for $10 \mathrm{~min}$. Antigen retrieval was performed by heating the sections at $95^{\circ} \mathrm{C}$ for $10 \mathrm{~min}$. Paraffin sections were incubated with anti-nestin (1:200), anti-DCX (1:200), anti-NF-H (1:200) at $4^{\circ} \mathrm{C}$ overnight and biotinylated secondary antibodies for $20 \mathrm{~min}$ at room temperature. The sections were incubated with avidin-biotin complex (ABC) (1:400) for another $20 \mathrm{~min}$, followed by washing with PBS three times, 5 min each time. The sections were counterstained with hematoxylin, followed by dehydration, and analyzed under light microscope. The antibodies are listed in Table I.

Western blot analysis. Animals were euthanized 4 weeks after MCAO using an overdose of anesthesia. Infarcted cerebral tissues were lysed with RIPA buffer containing protease/phosphatase inhibitors and phenylmethanesulfonyl fluoride (PMSF) on ice. After centrifugation at $15,000 \mathrm{rpm}$ for $15 \mathrm{~min}$, protein concentration was measured using a bicinchoninic acid (BCA) protein assay kit. Protein samples (30 $\mu \mathrm{g}$ each) were electrophoresed and transferred to polyvinylidene difluoride (PVDF) membrane, which was blocked with 5\% nonfat dry milk in PBS containing $0.2 \%$ Tween-20 $1.5 \mathrm{~h}$ at room temperature. After gentle shaking, and washing four times (10 min each) followed by incubation with primary antibodies: anti-nestin $(1: 1,000)$, anti-DCX $(1: 500)$, anti-NF-H $(1: 1,000)$ and anti- $\beta$-actin overnight at $4^{\circ} \mathrm{C}$, the membrane was incubated with horseradish peroxidase (HRP)-conjugated secondary antibodies $(1: 5,000)$ at room temperature for $1.5 \mathrm{~h}$. An ECL advance western blotting detection kit was used to detect chemiluminescence in the dark. The antibodies are listed in Table I.

$R T-P C R$. Total RNA was extracted from the right cerebral cortex with TRIzol (Invitrogen, Carlsbad, CA, USA). The cDNA synthesis was performed using the reverse transcription system with oligo $(\mathrm{dT})_{20}$ according to the manufacturer's instructions. A control reaction without the reverse transcriptase was also carried out. The amplifications were conducted on a real-time 
Table II. Primers used in quantitative RT-PCR.

\begin{tabular}{llcc}
\hline Primers & \multicolumn{1}{c}{ Sequences } & Product size & Manufacturer \\
\hline Nestin & $\begin{array}{l}\text { Forward: 5'-CTGGGCAAGTGGAACGTAGA-3' } \\
\text { Reverse: 5'-CCTCCCACCGCTGTTGAT-3' }\end{array}$ & \multirow{2}{*}{ 150 bp } & Invitrogen \\
DCX & $\begin{array}{l}\text { Forward: 5'-CCTCAGGGAGTGCGCTACAT-3' } \\
\text { Reverse: 5'-CGACCAGTTGGGATTGACATT-3' }\end{array}$ & $150 \mathrm{bp}$ & Invitrogen \\
NF-H & $\begin{array}{l}\text { Forward: 5'-GATGGCCCTGGATATTGAGA-3' } \\
\text { Reverse: 5'-TTCGCTTTTGACTTTTATGTGAG-3' }\end{array}$ & \multirow{2}{*}{$148 \mathrm{bp}$} & Invitrogen \\
& $\begin{array}{l}\text { Forward: 5'-TGTCACCAACTGGGACGATA-3' } \\
\text { Reverse: 5'-GGGGTGTTGAAGGTCTCAAA-3' }\end{array}$ & $165 \mathrm{bp}$ & Sangon Biotech \\
\hline
\end{tabular}

NF-H, neuronal specific filament; DCX, doublecortin.
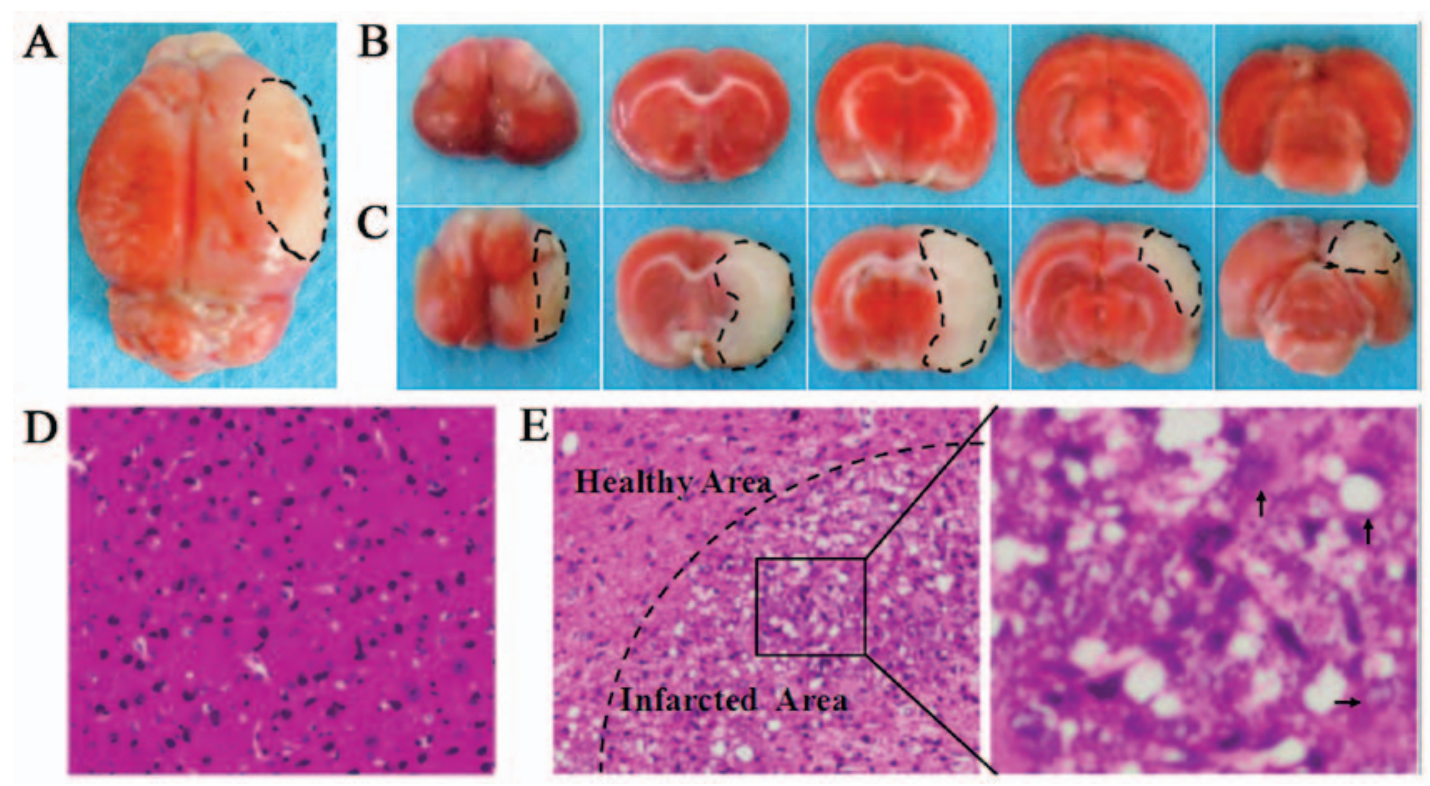

Figure 2. (A) The complete brain of middle cerebral artery occlusion (MCAO) models. (B) Serial coronal slices of sham-operated rats. (C) Consecutive coronal slices derived from MCAO models. 2,3,5-Triphenyltetrazolium chloride (TTC) staining showed red healthy zones and pale infarcted regions; Cerebral infarct volume was $\sim 32.1 \pm 1.8 \%$ in saline group. (D) H\&E staining of sham-operated animals revealed intact brain tissues, with uniform, round or oval neurons and pink cytoplasm with abundant and clear nucleus. No inflammatory cells were detected. (E) H\&E staining of MCAO models revealed lesions in the brain tissues, with diminished numbers of neurons and chaotic neuronal configuration. The cytoplasm was pale, eosinophilic and vacuolated. In addition, necrotic neurons with karyopyknosis, cellular gaps, debris and inflammatory cell infiltration were seen (x200).

PCR system (Bio-Rad, Hercules, CA, USA) using Accupower ${ }^{\circledR}$ 2X SYBR-Green I qPCR Master Mix (Bioneer, Daejeon, Korea) under the following conditions: pre-denaturation at $95^{\circ} \mathrm{C}$ for $15 \mathrm{~min}$, followed by 40 cycles of $95^{\circ} \mathrm{C}$ for $10 \mathrm{sec}, 53^{\circ} \mathrm{C}$ for $30 \mathrm{sec}$, and cooling at $4^{\circ} \mathrm{C}$ for $5 \mathrm{~min}$. The melting temperatures $(\mathrm{Tm})$ of the amplification products immediately after the last reaction cycle were used to determine the specificity. The $2^{-\triangle \Delta C T}$ method was used to compare the gene expression of neural markers in each group. All the PCRs were performed in triplicate and validated by the presence of a single peak in the melting curve. The primers are shown in Table II.

Statistical analysis. All data are expressed as mean \pm standard error of the mean (SEM). Means were compared using one-way ANOVA with Bonferroni's multiple comparison tests. $\mathrm{P}<0.05$ was considered statistically significant. GraphPad Prism version 5 was used for all the statistical analyses.

\section{Results}

Assessment of stroke. The transient MCAO is an established animal model of stroke. In the present study, the infarct zone ratio for each brain was calculated as follows: (left hemisphere area-right uninfarcted area)/(left hemisphere area*2), TTC staining showed that the ischemic areas accounted for an average $32.1 \pm 1.8 \%$ of the total volume at $24 \mathrm{~h}$ (Fig. 2). MCAO-induced injury mainly involves frontal, parietal, temporal, occipital and striatum regions of the right cerebral hemisphere. No visible lesions occurred in the sham-operated animals. H\&E staining revealed normal brain tissues in the sham-operated group, with uniform neuronal distribution. Neurons were round or oval, with pink cytoplasm, abundant and with clear nucleus. No inflammatory cells were detected. In the MCAO group, infarcted brain tissues were found, with a limited number of disordered neurons containing pale cyto- 
A

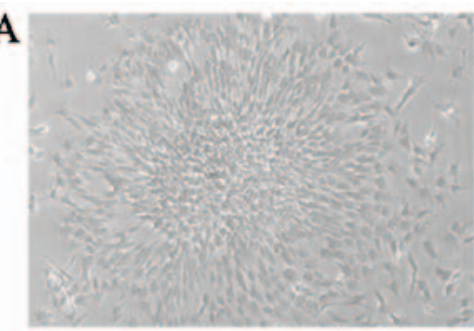

D

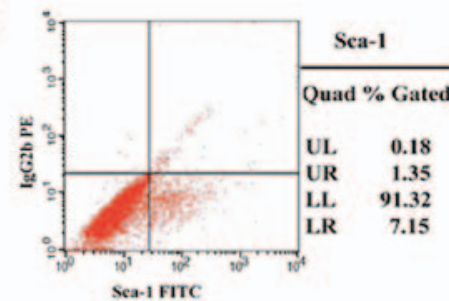

F
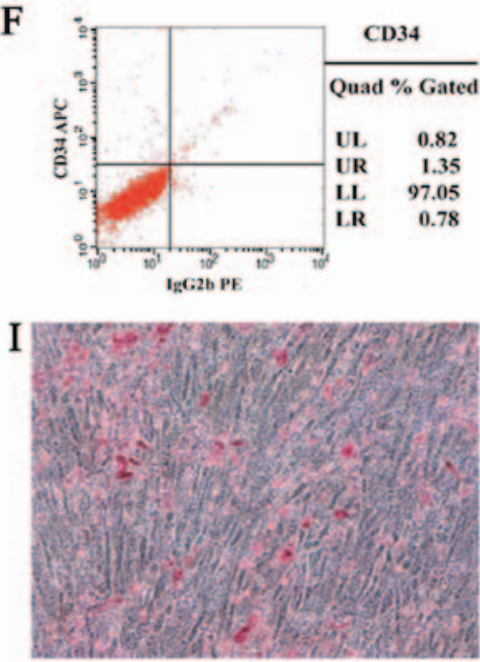

B

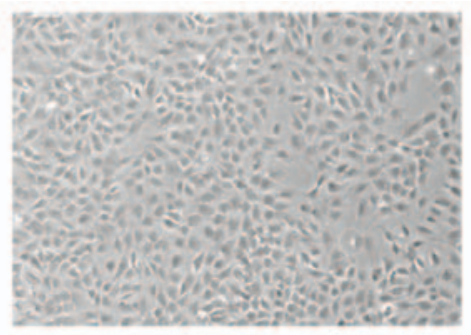

$\mathrm{E}$
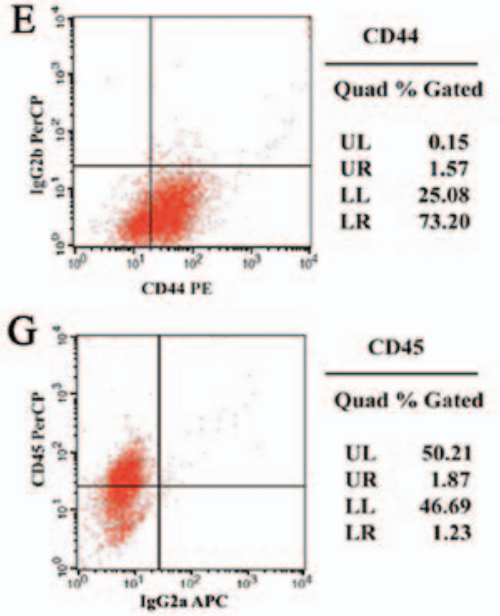

$\mathbf{J}$

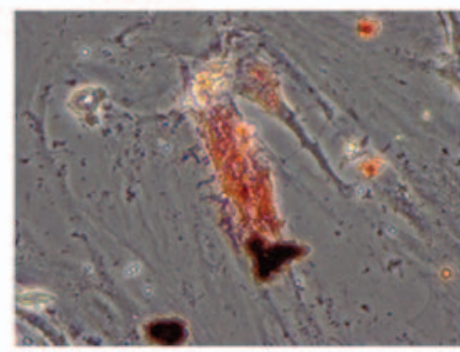

$\mathrm{C}$

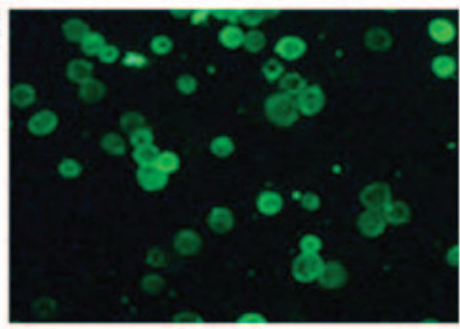

$\mathrm{H}$

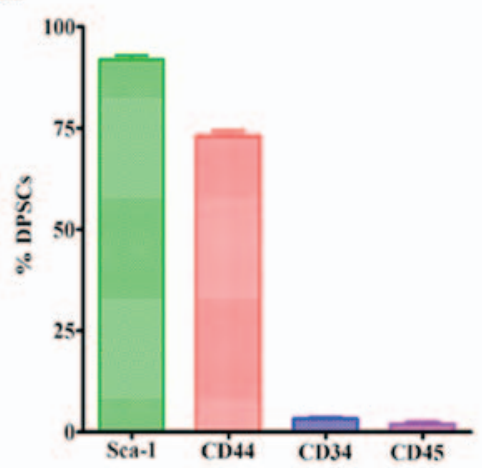

Figure 3. (A) P0 of dental pulp stem cells (DPSCs), showing plastic adherence and colony formation. (B) P3 of DPSCs with fibroblast-like morphology. (C) PKH67-labeled DPSCs emit green fluorescence under a fluorescent microscope at $488 \mathrm{~nm}$ (x40). (D-G) Fluorescence-activated cell sorting (FACS) of DPSCs. (H) Surface marker expression of the isolated DPSCs: $91.32 \%$ expressed Sca-1; 73.20\% expressed CD44; 1.35\% expressed CD34; and 1.23\% expressed CD45. (I-K) Multilineage differentiation of DPSC: mineralized nodules, calcium deposits and fat droplets (x100).

plasm, eosinophils and abundance of vacuoles. In addition, a large infiltration of necrotic neurons and inflammatory cells was observed. Widespread ischemic damage in the brain resulted in significant neurological deficit. The 18-point mNSS and the results of the adhesive removal tests increased significantly.

Characteristics of DPSCs. Cultured DPSCs exhibited plastic adherence, colony formation, fibroblast-like morphology, self-renewing capacity, expression of surface markers and multilineage differentiation (Fig. 3). Fluorescence-activated cell sorting (FACS) analyses were used to evaluate the expression of various surface markers on the isolated cells. In conclusion, $91.32 \%$ of the passage- 3 cells expressed stem cell marker Sca-1; and $73.20 \%$ expressed CD44. In contrast, few cells expressed hematopoietic stem cell markers, $1.35 \%$ expressed CD34; $1.23 \%$ expressed CD45. In addition, randomly distributed mineralized nodules were detected in the culture dish. Osteogenesis and adipogenesis were characterized by the presence of calcium deposits and fat droplets. In conclusion, the cells derived from SD rat dental pulp appeared to be largely DPSCs.
Migration and distribution of transplanted DPSCs. In the present study, we observed PKH67 fluorescent signal 3 weeks after DPSCs transplantation (Fig. 4). PKH67-labeled cells were abundant in the DPSC group around the periphery of the infarcted area, but not in the contralateral nonischemic brain (data not shown) under laser scanning confocal microscope. Several PKH67-labeled cells were found in the DPSCs+BDNF treatment group than in the DPSC group, as BDNF prolonged the viability of transplanted DPSCs. No PKH67-labeled cells were found in the brains of control rats. These results suggest that DPSCs penetrated through the blood-brain barrier (BBB) (23), proliferated, and migrated toward the ischemic areas of stroke. Homing ability is the characteristic of stem cells. Grafted cells can migrate and localize in injured areas of the brain tissues. PKH67 represents an ideal cell-labeling agent to track transplanted cells in vivo.

Expression of neural-specific markers. PKH67-labeled cells emitted green fluorescence at $488 \mathrm{~nm}$. Rhodamine-conjugated antibody emitted red fluorescence at $594 \mathrm{~nm}$, and DAPI displayed blue fluorescence at $405 \mathrm{~nm}$. We found that most of the PKH67-labeled DPSCs accumulated in the peripheral 


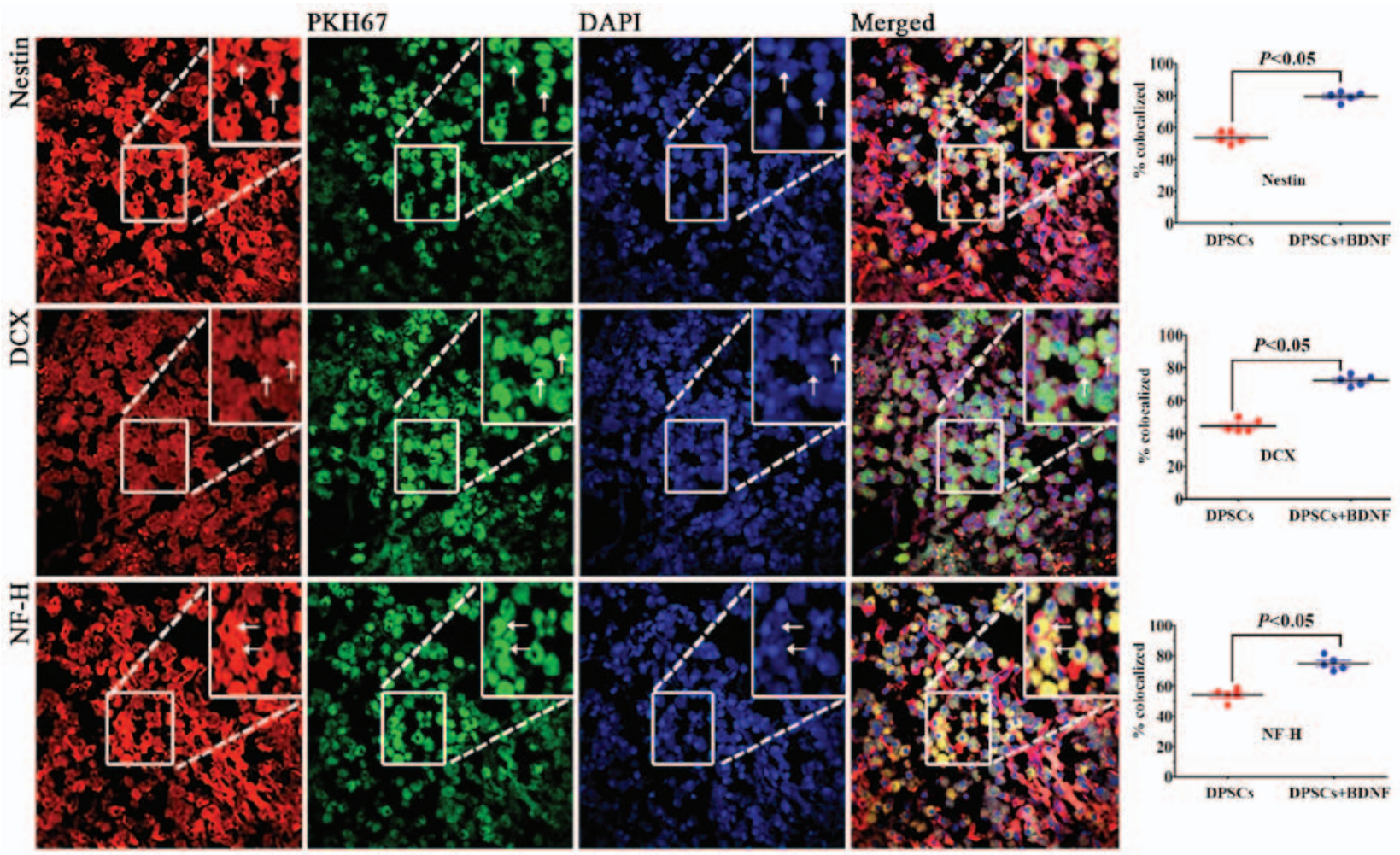

Figure 4. PKH67-labeled dental pulp stem cells (DPSCs) located in the peripheral infarcts or the infarct penumbra. Cells express neural-specific markers including nestin, doublecortin (DCX) and neuronal specific filament (NF-H). DAPI staining revealed blue fluorescence at $405 \mathrm{~nm}$. PKH67-labeled DPSCs were co-localized with a panel of neural-specific markers and DAPI. Positive cells were significantly higher in the DPSCs+brain-derived neurotrophic factor $(\mathrm{BDNF})$ group compared with the DPSCs group $(\mathrm{x} 400)(\mathrm{P}<0.05)$.
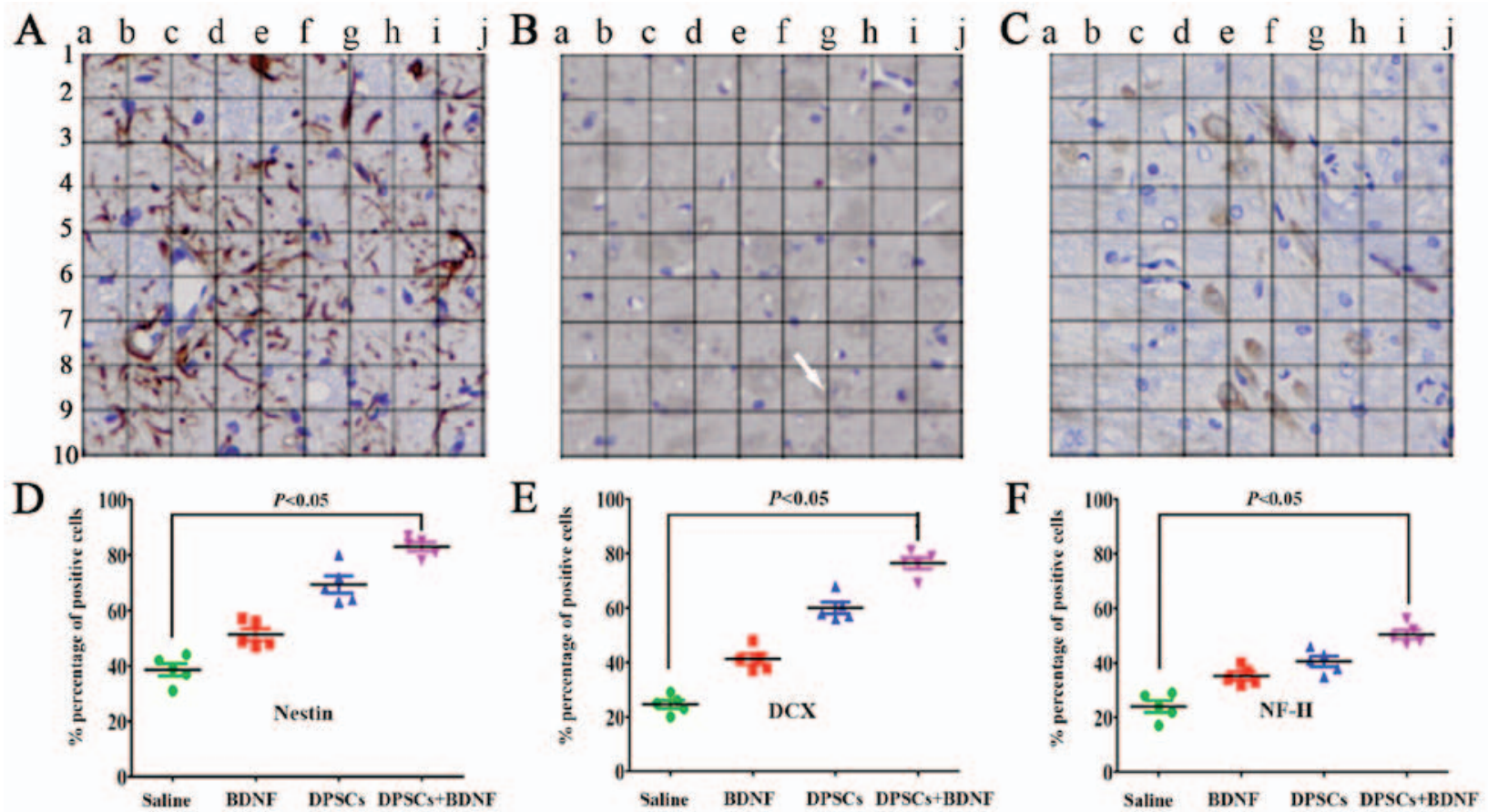

Figure 5. Positive cell numbers and percentages. (A-C) Nestin-, doublecortin (DCX)- and neuronal specific filament (NF-H)-immmunoreactive cells showing neuronal morphology around the infarcts. (D-F) Semi-quantitative data (x200). (B) DCX was localized mainly in the cytoplasma (arrow). The percentage of positive cells was significantly higher in the brain-derived neurotrophic factor (BDNF), dental pulp stem cells (DPSCs) and DPSCs+BDNF groups than in the saline group $(\mathrm{P}<0.05)$. Further, the percentage of positive cells in the DPSCs+BDNF group was significantly higher than in the BDNF and DPSCs groups $(\mathrm{P}<0.05)$.

ischemic regions of MCAO models (Fig. 4). They displayed neuronal morphology and were identified with a panel of neural-specific markers including nestin (24), DCX (25), NF-H and DAPI. The arrow in Fig. 5B shows that DCX 
A

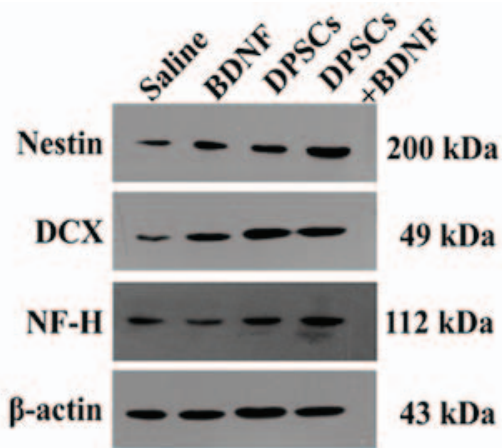

$\mathrm{C}$

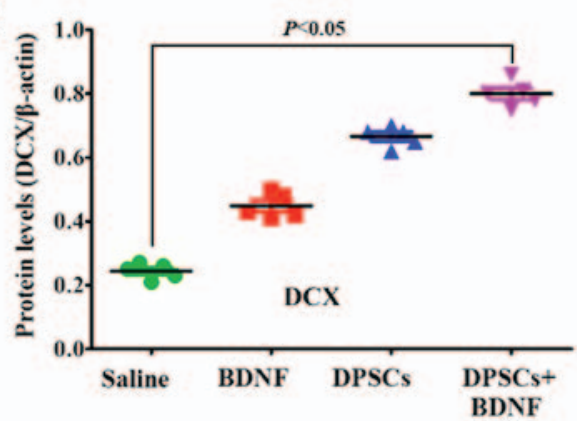

B

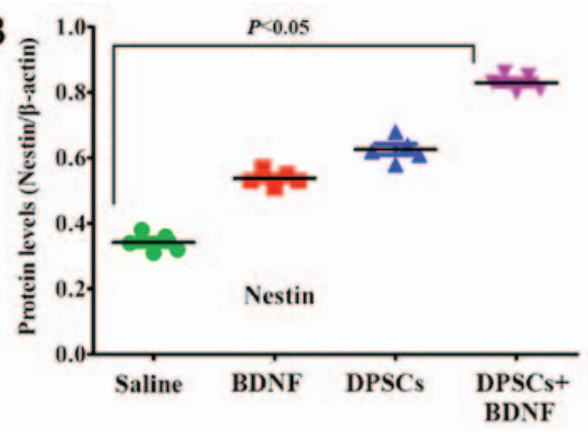

D

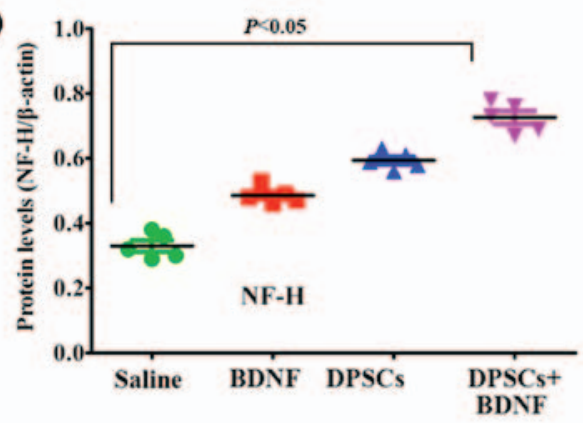

Figure 6. Western blot analysis of neuronal-specific markers. (A) Immunoreactive band. (B-D) Increased protein levels in the dental pulp stem cells (DPSCs)+brain-derived neurotrophic factor (BDNF) group compared with the other groups $(\mathrm{P}<0.05)$. Protein level in the DPSCs or BDNF group was significantly higher than in the saline control $(\mathrm{P}<0.05)$.

was localized mainly in the cytoplasma. We systematically analyzed and compared the expression of markers in each group. Immunofluorescence revealed increased number of positive cells in DPSCs+BDNF group compared with DPSCs group. The percentage of positive cells was significantly higher in the different treatment groups than in the saline control group $(\mathrm{P}<0.05)$. The number of positive cells in the DPSCs+BDNF group was significantly higher than in the BDNF and the DPSCs groups $(\mathrm{P}<0.05)$ (Fig. 5). Using western blot analysis and RT-PCR analysis, we further confirmed an increase in neuron-specific markers in the DPSCs+BDNF group compared with the other groups. Protein and gene markers in DPSCs or BDNF group were also higher than in the saline control group $(\mathrm{P}<0.05)$ (Figs. 6 and 7). Data suggest that BDNF promoted DPSC differentiation into neuron-like cells and triggered neurogenesis.

Weight comparison. The body weight of MCAO models decreased significantly $24 \mathrm{~h}$ after surgery and recovered slowly eventually. The weight was higher in the experimental groups than in the saline control group. The recovery in the DPSCs+BDNF group was significantly higher than in the BDNF and DPSCs groups $(\mathrm{P}<0.05)$. The groups differed significantly from each other suggesting that BDNF and DPSCs prevented neuronal ischemia (Fig. 8).

Treatment enhances sensorimotor functional recovery. The stroke models were functionally assessed using the adhesive-removal test and the mNSS on days 1, 7, 14, 21 and 28 after MCAO. All the animals showed functional impairment on day 1 postoperatively, followed by gradual recovery. Compared with the saline group, animals treated with BDNF or DPSCs exhibited improved neurological performance in the adhesive removal task and in mNSS at 1 week post-treatment $(\mathrm{P}<0.05)$. The combination treatment group showed greater functional recovery compared with other groups $(\mathrm{P}<0.05)$. The greatest improvement was observed in the DPSCs+BDNF-treated rats on day 28 post-MCAO $(\mathrm{P}<0.01)$. These data suggest that functional deficits following ischemia due to MCAO were successfully treated by intravenous transplantation of DPSCs or BDNF injection (Fig. 7). Further, DPSCs and BDNF showed synergistic neuroprotection against stroke.

\section{Discussion}

Induced pluripotent stem cells (26), embryonic stem cells (27), neural stem cells (28), bone marrow-derived mesenchymal stem cells (29) and DPSCs (30) exhibit neuroprotective effects in ischemic stroke animal models. DPSCs are easily harvested, cultured, amplified and cryo-preserved. They originate in the cranial neural crest and exhibit higher differentiation compared with other stem cells. Allogeneic stem cell transplantation may be used in clinical trials without the risk of immune rejection and ethical concerns. DPSCs represent ideal stem cells for treatment of neurological diseases. In the present study, we showed that: i) cells can be labeled with the green fluorescent dye PKH67 and tracked in vivo without cytotoxicity; ii) intravenously transplanted DPSCs retain their proliferative capacity in the peripheral ischemic regions of MCAO models; iii) PKH67labeled DPSCs were co-localized with neuronal markers and DAPI; iv) DPSC transplantation combined with BDNF injection enhanced the expression of neural differentiation markers including nestin, DCX, and NF-H, suggesting that BDNF promotes DPSC survival and differentiation into neuron-like cells; v) combination treatment with DPSCs and BDNF restores neurological function better than BDNF or DPSCs alone. 

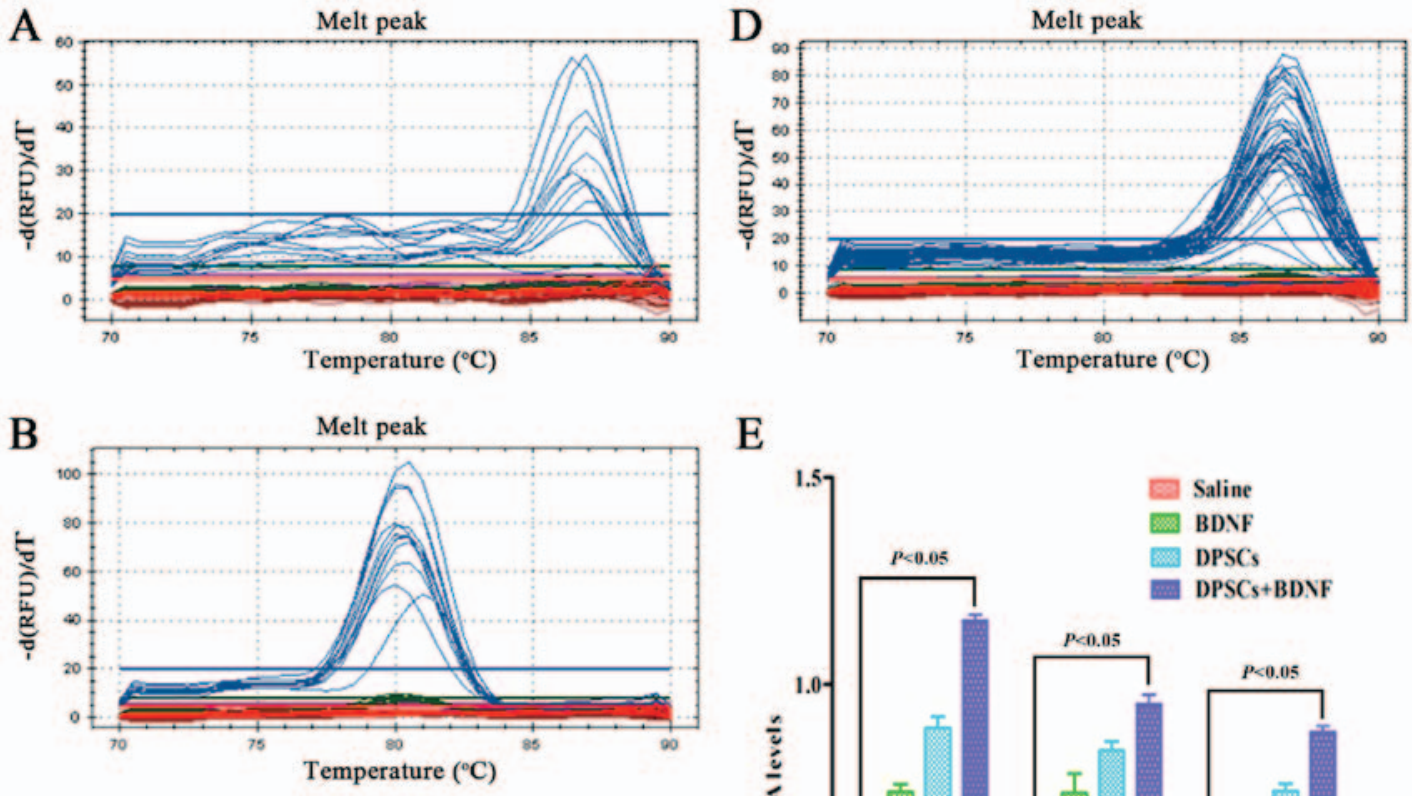

$\mathrm{E}$
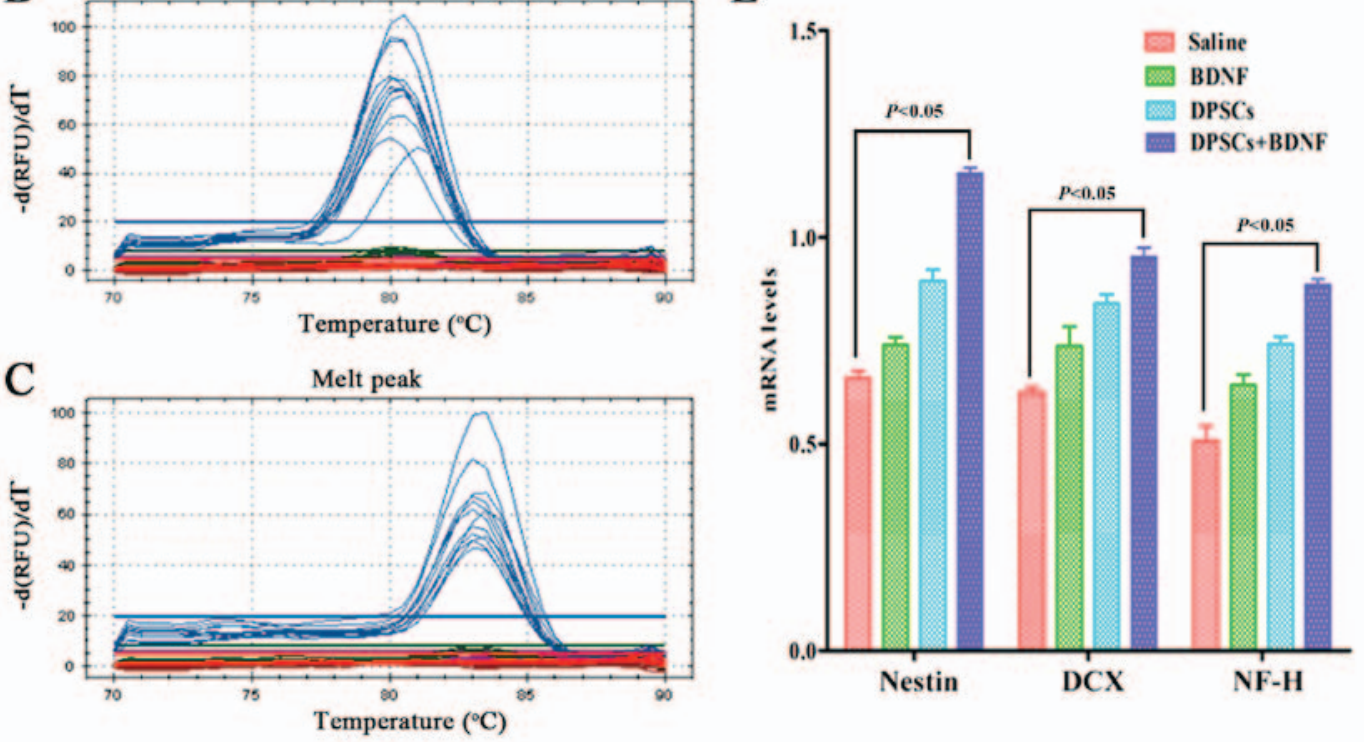

Figure 7. RT-PCR of gene expression of neuronal specific markers. (A-D) Melting curve of nestin, doublecortin (DCX), neuronal specific filament (NF-H) and $\beta$-actin. (E) mRNA levels of neural-specific markers in each group. Dental pulp stem cells (DPSCs)+brain-derived neurotrophic factor (BDNF) group gene expression was higher than in the other groups $(\mathrm{P}<0.05)$. Gene expression level in DPSCs or BDNF group was significantly higher than in the saline control group $(\mathrm{P}<0.05)$.
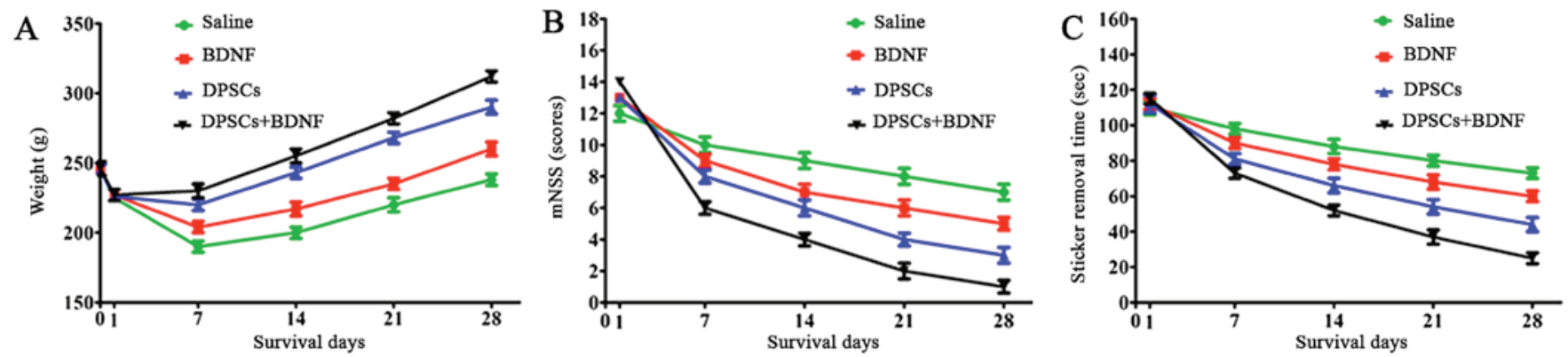

Figure 8. (A) Weight loss on day 1 after middle cerebral artery occlusion (MCAO) was reversed gradually. Significant differences were found between each group. (B) Modified neurological severity scores (mNSS). (C) Adhesive removal tests. All animals showed a remarkable functional deficit on day 1 after operation, followed by gradual recovery. Compared with the saline control, animals treated with brain-derived neurotrophic factor (BDNF) or dental pulp stem cells (DPSCs) showed better neurological performance in the adhesive removal task and mNSS at week 1 after treatment $(\mathrm{P}<0.05)$. The DPSCs+BDNF-treated group showed greater functional improvement compared with other groups $(\mathrm{P}<0.05)$. The greatest neurological improvements were observed in the DPSCs+BDNF-treated rats 4 weeks after MCAO $(\mathrm{P}<0.01)$.

MCAO is traditionally used to mimic ischemic stroke. TTC and H\&E staining revealed cerebral infarction in rat brains. Rats subjected to MCAO showed obvious weight loss and behavioral deficits postoperatively unlike the sham-operated rats. Our data indicated successful creation of focal cerebral ischemia models. We studied rat DPSCs displaying stem cell morphology, phenotype and multilineage differentiation potentials. Isolated DPSCs expressed mesenchymal stem cell markers Sca-1 and CD44, and rarely expressed hematopoietic or endothelial cell markers CD34 and CD45. Calcium deposition and fat droplets were found using Alizarin Red and Oil Red O staining, which revealed DPSC differentiation into bone and adipose cells. The green fluorescent dye PKH67 was used to label cell membrane and track transplanted stem cells in vivo. The high labeling efficiency suggested absence of cytotoxicity. Cells colocalized with green fluorescent, neural-specific markers and DAPI were observed in the peripheral ischemic regions under laser scanning confocal microscope, indicating DPSC survival in focal 
cerebral ischemia. According to previous experimental research and basic theory, stem cells have homing characteristic, grafted cells migrate and localize in injured areas in vivo. In the past, our group demonstrated similar results that transplanted bone marrow cells relocated to and resided mostly around the infarct penumbra in a mouse model of ischemic cerebral stroke (31). Many researchers have proved that stroke-induced stromal cell-derived factor-1 (SDF-1) and its receptor CXC chemokine receptor 4 (CXCR4) play a critical role in attracting transplanted cells into injured areas. Stroke-induced stromal cell derived factor-1 (SDF-1) may play a critical role in attracting transplanted cells to ischemic areas. Intravenously transplanted stem cells reach the site of injured area by interaction with SDF-1 and its CXC chemokine receptor 4 (CXCR4) (32-35). Intravenous delivery of stem cells is safe and effective without surgical trauma or clinical complications $(31,36)$. No adverse events were apparent after cellular transplantation or BDNF injection. DCX is expressed by neuronal precursor cells and is a biological marker for immature neurons. It is the 'gold standard' for evaluation of neurogenesis (25). Nestin, a marker of neural stem cells, is an intermediate filament protein synthesized during the early stages of neuronal development (24). NF-H is a mature neuronal marker. Engrafted DPSCs expressed neuralspecific markers including nestin, DCX and NF-H, indicating successful differentiation into neuron-like cells. Functionally differentiated cells integrate into neural networks and replace the damaged or lost neural tissues. Additional evidence is needed to corroborate the findings.

Animals in the BDNF, DPSCs and DPSCs+BDNF groups, showed better neurological performance than those in the saline group. Functional recovery occurs as a result of direct cell replacement. In addition, DPSCs secrete a series of neurotrophic factors, including glial-derived neurotrophic factor (GDNF), BDNF and NGF, which attenuate apoptosis and control neuronal survival, growth, and differentiation (37). Further, DPSCs modulate tissue microenvironment and alleviate inflammation. Central nervous system inflammation contributes to the pathogenesis of stroke and neurological deficits. Studies suggest that BDNF not only decreases local pro-inflammatory cytokines, but also increases the levels of anti-inflammatory mediators (38). Further, BDNF promotes angiogenesis and neurogenesis in MCAO rats. A test grid of frames was used to conduct semi-quantitative analysis of positive cells expressing nestin, DCX and NF-H (39). We found that the expression of nestin, DCX and NF-H was higher in the DPSCs+BDNF group than in the DPSCs. Expression of markers in the DPSCs or BDNF group was also higher than in the control group. BDNF injection may improve the viability and differentiation of transplanted DPSCs resulting in enhanced neuroprotection. The results suggest that combining BDNF and DPSCs had a synergistic protective effect against stroke, and represents an effective strategy to restore neurological function. The study provides experimental evidence supporting clinical investigation of cell and gene therapy in neurological diseases.

However, the study limitations relate to the short follow-up of the surviving DPSCs after transplantation in vivo. Further investigations are needed to elucidate the molecular mechanisms underlying the role of combined BDNF and DPSCs administration in cerebral ischemia.
In conclusion, notwithstanding the lack of established mechanisms underlying the role of stem cell therapies, the present study for the first time demonstrated that intravenous transplantation of rat-derived DPSCs promotes effective functional recovery in animal models of stroke without any adverse effect. BDNF administration may promote DPSC survival and differentiation into functional neurons. DPSC transplantation together with BDNF injection is a promising clinical strategy for the management of cerebral ischemia.

\section{Acknowledgements}

The authors thank the Laboratory Center of the Second Affiliated Hospital of Harbin Medical University for experimental and technical assistance.

\section{Funding}

This study was co-supported by the China Postdoctoral Science Foundation (no. 2012M520771) and the Heilongjiang Postdoctoral Fund (no. LBH-Z12152).

\section{Availability of data and material}

The datasets used and/or analyzed during the current study are available from the corresponding author on reasonable request.

\section{Authors' contributions}

JF designed the experiment and provided guidance. $\mathrm{XZ}$ and YZ implemented the experiment and were major contributors in writing the manuscript. XZ and YZ contributed equally. HL participated in the design of the experiment and performed the fluorescence-activated cell sorting (FACS) analyses. RW analyzed the immunofluorescence and immunohistochemistry. DY and BL performed the PCR and western blot analysis. All authors read and approved the final manuscript.

\section{Ethics approval and consent to participate}

The experimental design and procedures were conducted according to institutional guides for animal experiments approved by the Institutional Animal Care and Use Committees of Harbin Medical University.

\section{Consent for publication}

Not applicable.

\section{Competing interests}

The authors declare that they have no competing interests.

\section{References}

1. Grossman AW and Broderick JP: Advances and challenges in treatment and prevention of ischemic stroke. Ann Neurol 74: 363-372, 2013.

2. Gronthos S, Mankani M, Brahim J, Robey PG and Shi S: Postnatal human dental pulp stem cells (DPSCs) in vitro and in vivo. Proc Natl Acad Sci USA 97: 13625-13630, 2000. 
3. Nosrat IV, Widenfalk J, Olson L and Nosrat CA: Dental pulp cells produce neurotrophic factors, interact with trigeminal neurons in vitro, and rescue motoneurons after spinal cord injury. Dev Biol 238: 120-132, 2001.

4. Király M, Kádár K, Horváthy DB, Nardai P, Rácz GZ, Lacza Z, Varga $G$ and Gerber G: Integration of neuronally predifferentiated human dental pulp stem cells into rat brain in vivo. Neurochem Int 59: 371-381, 2011.

5. Fang CZ, Yang YJ, Wang QH, Yao Y, Zhang XY and He XH: Intraventricular injection of human dental pulp stem cells improves hypoxic-ischemic brain damage in neonatal rats. PLoS One 8: e66748, 2013.

6. Nosrat IV, Smith CA, Mullally P, Olson L and Nosrat CA: Dental pulp cells provide neurotrophic support for dopaminergic neurons and differentiate into neurons in vitro; implications for tissue engineering and repair in the nervous system. Eur J Neurosci 19: 2388-2398, 2004.

7. Apel C, Forlenza OV, de Paula VJ, Talib LL, Denecke B, Eduardo CP and Gattaz WF: The neuroprotective effect of dental pulp cells in models of Alzheimer's and Parkinson's disease. J Neural Transm (Vienna) 116: 71-78, 2009.

8. Lee HJ, Lim IJ, Lee MC and Kim SU: Human neural stem cells genetically modified to overexpress brain-derived neurotrophic factor promote functional recovery and neuroprotection in a mouse stroke model. J Neurosci Res 88: 3282-3294, 2010.

9. Han Q, Li B, Feng H, Xiao Z, Chen B, Zhao Y, Huang J and Dai J: The promotion of cerebral ischemia recovery in rats by laminin-binding BDNF. Biomaterials 32: 5077-5085, 2011.

10. Cotman $\mathrm{CW}$ and Berchtold NC: Exercise: A behavioral intervention to enhance brain health and plasticity. Trends Neurosci 25: 295-301, 2002.

11. Kokaia Z, Andsberg G, Yan Q and Lindvall O: Rapid alterations of BDNF protein levels in the rat brain after focal ischemia: Evidence for increased synthesis and anterograde axonal transport. Exp Neurol 154: 289-301, 1998.

12. SchäbitzWR,Schwab S,Spranger M and Hacke W: Intraventricular brain-derived neurotrophic factor reduces infarct size after focal cerebral ischemia in rats. J Cereb Blood Flow Metab 17: 500-506, 1997.

13. Yamashita K, Wiessner C, Lindholm D, Thoenen $\mathrm{H}$ and Hossmann KA: Post-occlusion treatment with BDNF reduces infarct size in a model of permanent occlusion of the middle cerebral artery in rat. Metab Brain Dis 12: 271-280, 1997.

14. Kurozumi K, Nakamura K, Tamiya T, Kawano Y, Kobune M, Hirai S, Uchida H, Sasaki K, Ito Y, Kato K, et al: BDNF genemodified mesenchymal stem cells promote functional recovery and reduce infarct size in the rat middle cerebral artery occlusion model. Mol Ther 9: 189-197, 2004

15. Nomura T, Honmou O, Harada K, Houkin K, Hamada $\mathrm{H}$ and Kocsis JD: I.V. infusion of brain-derived neurotrophic factor gene-modified human mesenchymal stem cells protects against injury in a cerebral ischemia model in adult rat. Neuroscience 136: 161-169, 2005.

16. Chang DJ, Lee N, Choi C, Jeon I, Oh SH, Shin DA, Hwang TS, Lee HJ, Kim SU, Moon H, et al: Therapeutic effect of BDNFoverexpressing human neural stem cells (HB1.F3.BDNF) in a rodent model of middle cerebral artery occlusion. Cell Transplant 22: 1441-1452, 2013.

17. Ellis KM, O'Carroll DC, Lewis MD, Rychkov GY and Koblar SA: Neurogenic potential of dental pulp stem cells isolated from murine incisors. Stem Cell Res Ther 5: 30, 2014

18. Huang XF, Yuan SJ and Yang C: Effects of total flavonoids from Drynaria fortunei on the proliferation and osteogenic differentiation of rat dental pulp stem cells. Mol Med Rep 6: 547-552, 2012.

19. Hata M, Omi M, Kobayashi Y, Nakamura N, Tosaki T, Miyabe M, Kojima N, Kubo K, Ozawa S, Maeda H, et al: Transplantation of cultured dental pulp stem cells into the skeletal muscles ameliorated diabetic polyneuropathy: Therapeutic plausibility of freshly isolated and cryopreserved dental pulp stem cells. Stem Cell Res Ther 6: 162, 2015.

20. Longa EZ, Weinstein PR, Carlson S and Cummins R: Reversible middle cerebral artery occlusion without craniectomy in rats. Stroke 20: 84-91, 1989.

21. Chen J, Li Y, Wang L, Zhang Z, Lu D, Lu M and Chopp M: Therapeutic benefit of intravenous administration of bone marrow stromal cells after cerebral ischemia in rats. Stroke 32: 1005-1011, 2001.
22. Reglodi D, Tamás A and Lengvári I: Examination of sensorimotor performance following middle cerebral artery occlusion in rats. Brain Res Bull 59: 459-466, 2003.

23. Winderlich JN, Kremer KL and Koblar SA: Adult human dental pulp stem cells promote blood-brain barrier permeability through vascular endothelial growth factor- $\alpha$ expression. J Cereb Blood Flow Metab 36: 1087-1097, 2016.

24. Michalczyk K and Ziman M: Nestin structure and predicted function in cellular cytoskeletal organisation. Histol Histopathol 20: 665-671,2005.

25. Couillard-Despres S, Winner B, Schaubeck S, Aigner R, Vroemen M, Weidner N, Bogdahn U, Winkler J, Kuhn HG and Aigner L: Doublecortin expression levels in adult brain reflect neurogenesis. Eur J Neurosci 21: 1-14, 2005.

26. Chen SJ, Chang CM, Tsai SK, Chang YL, Chou SJ, Huang SS, Tai LK, Chen YC, Ku HH, Li HY, et al: Functional improvement of focal cerebral ischemia injury by subdural transplantation of induced pluripotent stem cells with fibrin glue. Stem Cells Dev 19: 1757-1767, 2010.

27. Tae-Hoon L and Yoon-Seok L: Transplantation of mouse embryonic stem cell after middle cerebral artery occlusion. Acta Cir Bras 27: 333-339, 2012.

28. Huang L, Wong S, Snyder EY, Hamblin MH and Lee JP: Human neural stem cells rapidly ameliorate symptomatic inflammation in early-stage ischemic-reperfusion cerebral injury. Stem Cell Res Ther 5: 129, 2014

29. Mitkari B, Kerkelä E, Nystedt J, Korhonen M, Mikkonen V, Huhtala $\mathrm{T}$ and Jolkkonen J: Intra-arterial infusion of human bone marrow-derived mesenchymal stem cells results in transient localization in the brain after cerebral ischemia in rats. Exp Neurol 239: 158-162, 2013

30. Yamagata M, Yamamoto A, Kako E, Kaneko N, Matsubara K, Sakai K, Sawamoto K and Ueda M: Human dental pulp-derived stem cells protect against hypoxic-ischemic brain injury in neonatal mice. Stroke 44: 551-4, 2013

31. Zhang XM, Du F, Yang D, Yu CJ, Huang XN, Liu W and Fu J: Transplanted bone marrow stem cells relocate to infarct penumbra and co-express endogenous proliferative and immature neuronal markers in a mouse model of ischemic cerebral stroke. BMC Neurosci 11: 138, 2010.

32. Rosenkranz K, Kumbruch S, Lebermann K, Marschner K, Jensen A, Dermietzel R and Meier C: The chemokine SDF-1/CXCL12 contributes to the 'homing' of umbilical cord blood cells to a hypoxic-ischemic lesion in the rat brain J Neurosci Res 88: 1223-1233, 2010.

33. Miller JT, Bartley JH, Wimborne HJ, Walker AL, Hess DC, Hill WD and Carroll JE: The neuroblast and angioblast chemotaxic factor SDF-1 (CXCL12) expression is briefly up regulated by reactive astrocytes in brain following neonatal hypoxic-ischemic injury. BMC Neurosci 6: 63, 2005.

34. Hill WD, Hess DC, Martin-Studdard A, Carothers JJ, Zheng J, Hale D, Maeda M, Fagan SC, Carroll JE and Conway SJ: SDF-1 (CXCL12) is upregulated in the ischemic penumbra following stroke: Association with bone marrow cell homing to injury. J Neuropathol Exp Neurol 63: 84-96, 2004.

35. Li M, Sun X, Ma L, Jin L, Zhang W, Xiao M and Yu Q: SDF-1/CXCR4 axis induces human dental pulp stem cell migration through FAK/PI3K/Akt and GSK3 $\beta / \beta$-catenin pathways. Sci Rep 7: 40161, 2017.

36. Zhang XM, Du F, Yang D, Wang R, Yu CJ, Huang XN, Hu HY, Liu W and Fu J: Granulocyte colony-stimulating factor increases the therapeutic efficacy of bone marrow mononuclear cell transplantation in cerebral ischemia in mice. BMC Neurosci 12: 61, 2011.

37. Kvinnsland IH, Luukko K, Fristad I, Kettunen P, Jackson DL, Fjeld K, von Bartheld CS and Byers MR: Glial cell line-derived neurotrophic factor (GDNF) from adult rat tooth serves a distinct population of large-sized trigeminal neurons. Eur J Neurosci 19: 2089-2098, 2004.

38. Jiang Y, Wei N, Zhu J, Lu T, Chen Z, Xu G and Liu X: Effects of brain-derived neurotrophic factor on local inflammation in experimental stroke of rat. Mediators Inflamm 2010: 372423, 2010.

39. Melvin NR and Sutherland RJ: Quantitative caveats of standard immunohistochemical procedures: Implications for optical disector-based designs. J Histochem Cytochem 58: 577-584, 2010.

This work is licensed under a Creative Commons Attribution-NonCommercial-NoDerivatives 4.0 International (CC BY-NC-ND 4.0) License. 